\title{
A New scoring system for colposcopy for detection Cervical Intraepithelial Neoplasia 2+
}

\author{
V. Villoslada ${ }^{1}$, C. Acosta ${ }^{2}$, B. Fora ${ }^{3}$, M. Alvarez ${ }^{4}$
}

1 National institute of neoplastic diseases, Dept of Gynaecology Surgery, Lima, Perú. Data analyst, visiting professor and researcher of the School of Human Medicine, National University of Cajamarca, Perú.

2 Bachelors' in biology and data analyst.

3 Obstetrics and Gynecology, Hipolito Unanue Hospital, Tacna, Perú.

1 National institute of neoplastic diseases, Dept of Gynaecology Surgery, Lima, Perú.

Background and Aims:

To construct a new scoring system for colposcopic examination that can better prediction for detection Cervical Intraepithelial Neoplasia $2+$ and facilitate education of colposcopists.

Methods:

Six hundred and fifty patients referred for colposcopy were examined. Four variables were scored: aceto whiteness, borders, vessels, and staining time from the regression coefficients. And finally the respective roc curves are constructed with the total of the variables and the different combinations. Hypothesis testing is done to see which curve is the best.

Results:

The score to use is summarized in table 1 . The analysis with the new score showed good sensitivity and specificity see Table 2 . The replacement of values by the coefficients obtained from each variable gave a more useful and simple scoring system. To choose the best score, AUC graphs were made comparing the different scores obtained by the combinations of the variables see graph 1 .

Conclusions:

The new scoring only needs two variables (Aceto whiteness plus borders) and the best cutoff point is 7 with a Se $95 \%$, Es $87 \%$, PPV $94 \%$, NPV $88 \%$

\begin{tabular}{llcc}
\multicolumn{2}{l}{ Table 1. Scores used to calculate the new Score } & \multicolumn{2}{l}{ Score } \\
\hline \multicolumn{2}{c}{ Aceto whiteness } & Coef. Beta & \\
& dense & 5.7842 & 6 \\
& Fain & 1.4186 & 1 \\
& Absent & 1 & 0 \\
\hline Borders & & & \\
& Defined & 6.9654 & 7 \\
& little defined & 3.9751 & 4 \\
& Absent & 1 & 0 \\
\hline Vessels & & & \\
& Thick & 1.457 & 1 \\
& thin & 0.4859 & 1 \\
& Absent & 1 & 0 \\
\hline Staining time & & & \\
& fast & 1.33702 & 1 \\
& Slow & 1 & 0 \\
\hline
\end{tabular}

Coef. Beta: binary logistic regression coefficient

\begin{tabular}{|c|c|c|c|c|c|c|}
\hline \multirow{2}{*}{ Tabla 2.} & \multirow{2}{*}{$\frac{\text { arameters of }}{\text { Total }}$} & \multirow{2}{*}{$\begin{array}{r}\text { area } \\
\text { Color }\end{array}$} & \multirow{2}{*}{$\begin{array}{l}\text { under } \\
\text { borders }\end{array}$} & \multicolumn{3}{|l|}{ the curve } \\
\hline & & & & Color/borders $\quad \mathrm{c}$ & Color/borders/vessels & Borders/vessels \\
\hline $\begin{array}{l}\text { AUC (IC } \\
95 \%)\end{array}$ & $\begin{array}{c}0.946(0.927 \\
0.966)\end{array}$ & $0.913(0.889,0.938)$ & $0.867(0.8410 .892$ & $\begin{array}{cc} & 0.947 \\
392) & 0.9280 .966\end{array}$ & 56) $0.945(0.925$, & $\begin{array}{c}0.868(0.84, \\
0.896)\end{array}$ \\
\hline cutoff & 9 & 6 & 4 & 7 & 9 & 4 \\
\hline Se & 0.94 & 0.93 & 1.00 & 0.95 & 0.94 & 1.00 \\
\hline Es & 0.88 & 0.88 & 0.39 & 0.87 & 0.88 & 0.39 \\
\hline PPV & 0.94 & 0.95 & 0.78 & 0.94 & 0.95 & 0.78 \\
\hline NPV & 0.88 & 0.86 & 0.98 & 0.88 & 0.87 & 0.98 \\
\hline LR+ & 7.63 & 7.85 & 1.63 & 7.35 & 7.93 & 1.63 \\
\hline LR- & 0.06 & 0.08 & 0.01 & 0.06 & 0.07 & 0.01 \\
\hline
\end{tabular}

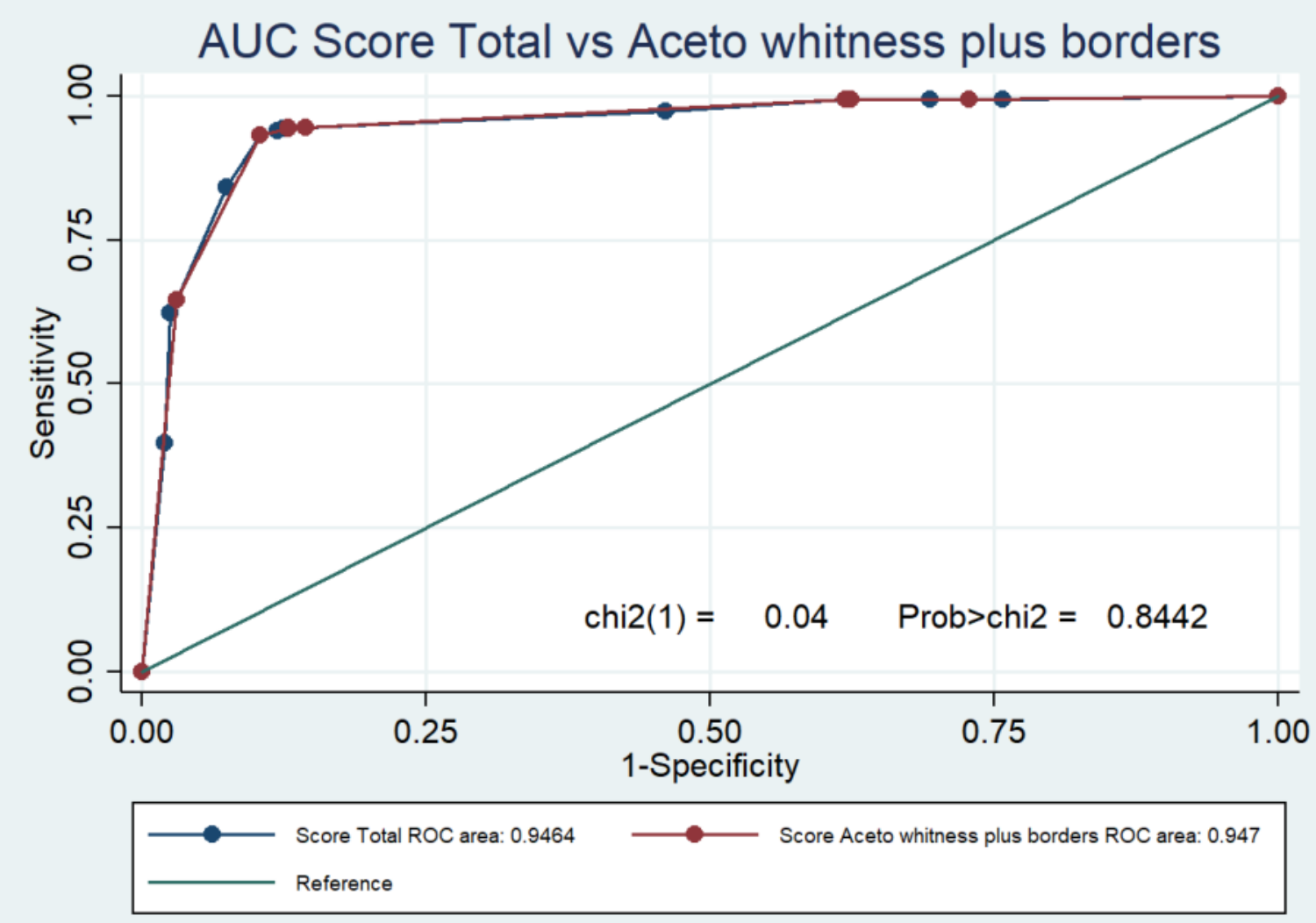

